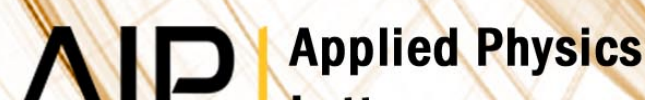 \\ Letters
}

\section{Confinement effects on the low temperature magnetic structure of MnP nanocrystals}

A. de Andrés, R. Ramírez-Jiménez, M. García-Hernández, S. Lambert-Milot, and R. A. Masut

Citation: Appl. Phys. Lett. 99, 182506 (2011); doi: 10.1063/1.3658393

View online: http://dx.doi.org/10.1063/1.3658393

View Table of Contents: http://apl.aip.org/resource/1/APPLAB/v99/i18

Published by the American Institute of Physics.

\section{Additional information on Appl. Phys. Lett.}

Journal Homepage: http://apl.aip.org/

Journal Information: http://apl.aip.org/about/about_the_journal

Top downloads: http://apl.aip.org/features/most_downloaded

Information for Authors: http://apl.aip.org/authors

\section{ADVERTISEMENT}

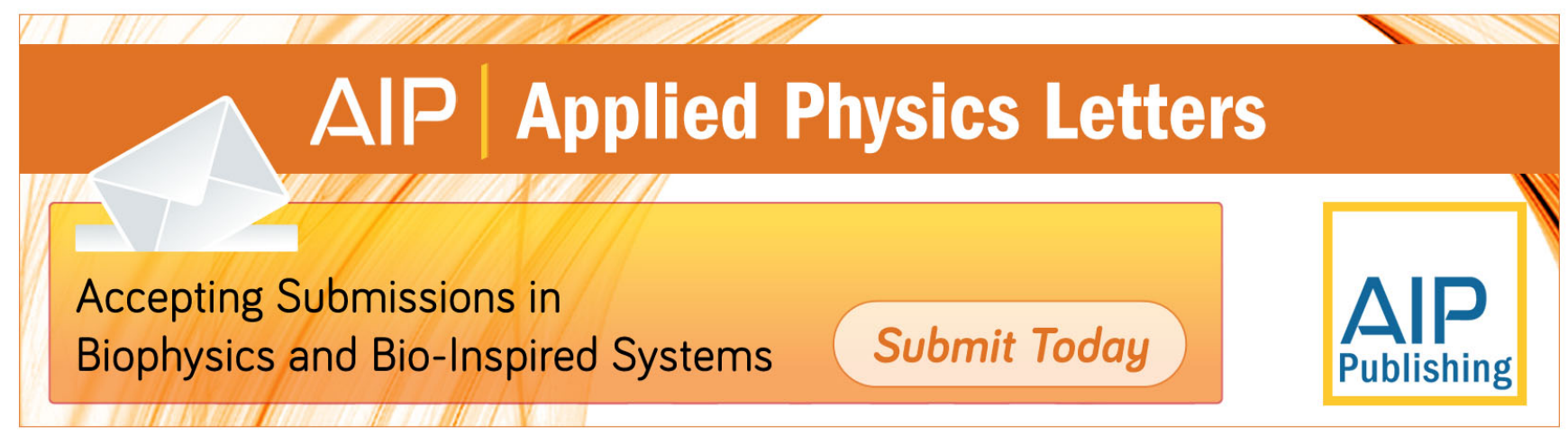




\title{
Confinement effects on the low temperature magnetic structure of $\mathrm{MnP}$ nanocrystals
}

\author{
A. de Andrés, ${ }^{1, a)}$ R. Ramírez-Jiménez, ${ }^{2}$ M. García-Hernández, ${ }^{1}$ S. Lambert-Milot, ${ }^{3}$ \\ and R. A. Masut ${ }^{3}$ \\ ${ }^{1}$ Instituto de Ciencia de Materiales de Madrid, CSIC, Cantoblanco, 28049 Madrid, Spain \\ ${ }^{2}$ Departamento de Física, Escuela Politécnica Superior, Universidad Carlos III de Madrid, \\ Avenida Universidad 30, 28911 Madrid, Spain \\ ${ }^{3}$ Regroupement québécois sur les matériaux de pointe (RQMP) and Département de génie physique, \\ École Polytechnique de Montréal, CP 6079 Succ. Centre-ville, Montreal, Quebec H3C 3A7, Canada
}

(Received 20 July 2011; accepted 13 October 2011; published online 2 November 2011)

\begin{abstract}
The low temperature magnetic properties of $\mathrm{MnP}$ nanocrystals $(15-40 \mathrm{~nm})$, both in GaP:MnP epilayers and $\mathrm{MnP}$ films, are significantly different compared to bulk and cannot apparently be explained by differences in the structure. A simple model of localized spins is used to describe the magnetic screw structure confined to nanocrystals. The results indicate that the observed magnetic behaviour is related to the nanometric size and to changes in the coupling constants most probably localized at an external grain shell. The nucleation of helical regions at the surface of the ferromagnetic grains is proposed as a possible mechanism for the reversal of the magnetization. (C) 2011 American Institute of Physics. [doi:10.1063/1.3658393]
\end{abstract}

Magnetic semiconductors with high ferromagnetic transition temperatures $\left(\mathrm{T}_{\mathrm{C}}\right)$ and spin polarized carriers are the paradigm for spintronics, however, so far, III-V based materials present low $\mathrm{T}_{\mathrm{C}}$ and metal transition doped oxides, with high $\mathrm{T}_{\mathrm{C}}$ 's, are still not well controlled, many times with non homogeneous dopant distribution forming nanosized metastable phases in the matrix..$^{1-3}$ On the other hand, Mn-based submicron ferromagnets embedded in III-V semiconductor host layers offer interesting properties for magnetoelectronic devices and can in principle be tailored to exhibit room-temperature ferromagnetic behaviour as well as giant magneto-optical Kerr and Faraday effects. ${ }^{1,4,5}$ Therefore, in order to obtain promising functionalities and devices, we focussed on the combination of MnP, a RT ferromagnet, and $\mathrm{GaP}$, a wide indirect gap semiconductor, which are nearly lattice-matched to Si. Here, we present and discuss the magnetic properties of MnP nanocrystals in two different situations: embedded in GaP epilayers and as polycrystalline thin films, both grown by metal organic vapour phase epitaxy (MOVPE) on GaP (001). Previous detailed structural characterizations established that MnP nanocrystals $(15-20 \mathrm{~nm}$ in diameter) are embedded in GaP:Mn epilayers that are semicoherent with the substrate. ${ }^{6}$ The grains of the studied MnP film have around $40 \mathrm{~nm}$ and lattice parameters within $0.2 \%$ of bulk MnP. ${ }^{7}$ Since the local structure and symmetry of Mn in $\mathrm{MnP}$ nanocrystals are found to be similar to those of bulk crystals and strain effects seem hardly compatible with the reported variations of both transition temperatures $T_{C}$ and $\mathrm{T}_{\mathrm{N}}{ }^{7}$ it becomes important to determine what is at the origin of the markedly different magnetic behavior of the nanocrystals, particularly at low temperatures. A simple model including ferro- $\left(J_{1}\right)$ and antiferro-magnetic (AFM) $\left(J_{2}\right)$ interactions is further developed here to evaluate the effects of nanometric size on the magnetic structure of $\mathrm{MnP}$.

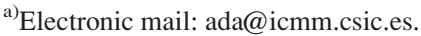

Bulk $\mathrm{MnP}$ crystallizes in the $\mathrm{Pbnm}$ space group $(a=5.918 \AA>b=5.258 \AA>c=3.172 \AA \quad($ Ref. 8)] $)$ and presents a strong biaxial magneto-crystalline anisotropy with its longer axis $(a)$ as the harder magnetic axis, and the shorter (c), the softer. $\mathrm{MnP}$ is ferromagnetic below $\mathrm{T}_{\mathrm{C}}=291.5 \mathrm{~K}$, hellimagnetic (screw) below $\mathrm{T}_{\mathrm{N}}=47 \mathrm{~K}$ and shows field induced transitions. ${ }^{9,10}$ At $4 \mathrm{~K}$, a full rotation of the magnetic moment is completed each $9 a$ (about $5 \mathrm{~nm}$ ). ${ }^{11} \mathrm{GaP}: \mathrm{MnP}$ films, around $300 \mathrm{~nm}$ thick, were grown on $\mathrm{GaP}(001)$ substrates in a low-pressure cold-wall MOVPE reactor ${ }^{7}$ at $650^{\circ} \mathrm{C}$. MnP films were grown under similar conditions, also on $\mathrm{GaP}(001)$. The MnP powder was commercially obtained from Alfa Aesar, whose average grain size is $150 \mu \mathrm{m}$. The magnetic characterization was achieved as a function of temperature $(2-350 \mathrm{~K})$ and magnetic field (up to $5 \mathrm{~T}$ ) with a superconducting quantum interference device magnetometer from Quantum Design.

Fig. 1 shows the large shift to higher temperatures of $T_{N}$ (from $47 \mathrm{~K}$ for bulk to $82 \mathrm{~K}$ for MnP:GaP) while $\mathrm{T}_{\mathrm{C}}$ changes slightly (see derivatives in Fig. 1(c)). Also, while the field cooled (FC) magnetization in the screw phase is almost zero in bulk MnP, in MnP film, and in nanocrystals, the magnetization remains significant. Figs. 1(d) and 1(e) evidence the similarities between the films. Both show high coercive fields $\left(\mathrm{H}_{\mathrm{C}}\right)$ in the screw and collinear ferromagnetic phases as well as high remanence in contrast to the bulk $\left(\mathrm{H}_{\mathrm{C}} \simeq 0\right.$ and no remanence, in powder or single crystal $^{7,9}$ ).

To study the effect of the nanocrystals finite size, we calculated the ground state magnetic structure for a slice of $\mathrm{MnP}$ with its finite length along the screw axis $a$ (size $=\mathrm{Na} / 2$, where $N=$ number of planes perpendicular to $a$ ) using the Hamiltonian and parameters from Ref. 13. A 1D model along the hard $(a)$ axis can be justified because the major effect of confinement occurs along this axis, while confinement in the two perpendicular directions ( $b$ and $c$ axes) is expected to be relevant on the smaller scale of the Mn-Mn distance $(\sim 0.3 \mathrm{~nm})$ and, thus, can be ignored for the $15-40 \mathrm{~nm}$ clusters 


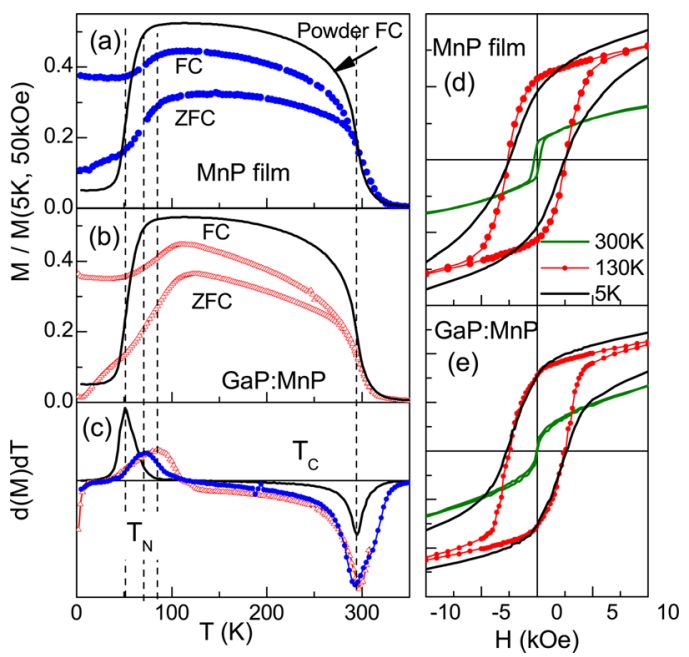

FIG. 1. (Color online) FC and ZFC Magnetizations measured at $1 \mathrm{kOe}$, normalized to the values at $5 \mathrm{~K}$ and $50 \mathrm{kOe}$, of (a) MnP film, (b) GaP:MnP, and for MnP powder (black line). (c) Magnetization derivatives showing the two magnetic transitions. Magnetization cycles at $5 \mathrm{~K}, 130 \mathrm{~K}$, and $300 \mathrm{~K}$ of (d) MnP film and (e) GaP:MnP epilayer.

we are considering here. The magnetic ground state of bulk $\mathrm{MnP}$ was calculated by considering localized spins on $\mathrm{Mn}$, which are coupled FM within the planes perpendicular to the hard $a$ axis. The different planes have equivalent spins $S_{n}$ whose directions are fitted to minimize the magnetic energy. The exchange coupling of each layer with its nearest neighbour is FM $\left(J_{1}\right)$, while it is AFM with its next nearest layer $\left(J_{2}\right) .{ }^{12}$ In the absence of anisotropy, the ratio $J_{2} / J_{1}$ determines whether the ground state is FM or screw (if $J_{2} / J_{1}>1 / 4$ ). The ground state for an infinite $\mathrm{MnP}$ crystal is the screw phase with an angle between the magnetic moments of two successive planes $\alpha=21^{\circ},{ }^{13}$ in agreement with experimental results. $^{11}$

The helical structure varies with the crystal size $(\mathrm{Na} / 2)$, as determined by energy minimization. Fig. 2(a) presents the evolution of $\alpha$ along the $a$ direction for two grain sizes $(\mathrm{N}=60, \mathrm{~N}=25$ ), showing the drastic reduction of $\alpha$ for the 5-6 planes near each surface. Therefore, the deviation from the uniform helical bulk structure is more important as the size is reduced. Fig. 2(b) represents the magnetic moments of the planes for two grains with similar number of planes $\mathrm{N}=28$ and $\mathrm{N}=32$. The total resulting moment is different

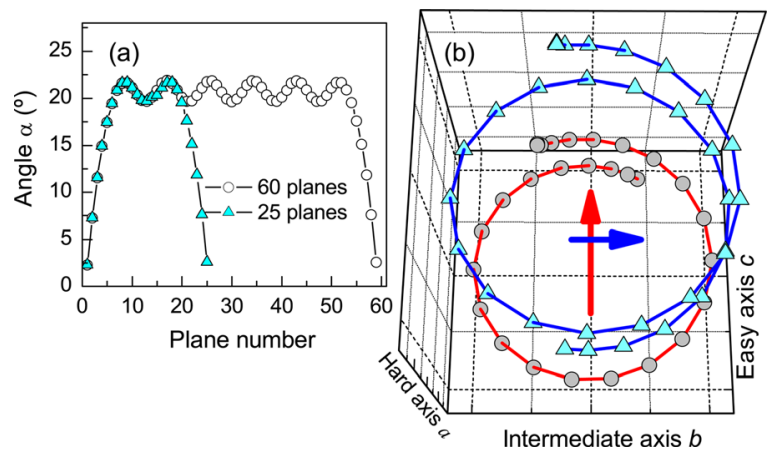

FIG. 2. (Color online) (a) Calculated angles between the magnetic moments of two successive planes $(\alpha)$ in grains with $\mathrm{N}=25(7.4 \mathrm{~nm})$ and $60(18 \mathrm{~nm})$ along their hard $a$ axis. (b) 3D representations of the ends of the magnetic moment vectors for each plane in two cases $\mathrm{N}=28$ (circles) and $\mathrm{N}=32$ (triangles). The arrows indicate the directions of the resulting moments of the whole grain (vertical $\mathrm{N}=28(8.3 \mathrm{~nm})$ and horizontal $\mathrm{N}=32(9.5 \mathrm{~nm})$ ). from zero (contrary to what occurs for an infinite screw phase) and switches from the easy axis, $c$, to the intermediate axis, $b$, depending on the size of the nanocrystals, due to the magnetic anisotropy. Fig. 3 represents the total magnetization (normalized to the fully ferromagnetic value) versus the number of planes along the hard direction. Both components, in the $b c$ plane, are plotted in the upper panel. The direction of the whole grain magnetization switches between $b$ and $c$ axes as the size increases. The range of sizes of the MnP nanocrystals in GaP and grains in the MnP film is indicated with horizontal arrows in Fig. 3(b).

These calculations also indicate that the magnetization of nanocrystals is finite and significant (between $5 \%$ and $10 \%$ of that of a collinear ferromagnetic magnetization) and explain the measured FC and zero field cooled (ZFC) magnetization curves; after $\mathrm{ZFC}$, the different grains have a net magnetic moment with a random distribution along both senses of $b$ or $c$ axes so that the measured moment is almost zero (Fig. 1, ZFC curves). When the samples are FC, the moments align with the applied field (Fig. 1, FC) and result in a non-zero magnetic moment in the $b c$ plane (Fig. 3). FC and ZFC curves differ up to about $\mathrm{T}_{\mathrm{C}}$ due to the high coercive fields of the samples $(2-2.7 \mathrm{kOe})$ compared to the applied field, $1 \mathrm{kOe}$. The calculated net moment per plane increases as the crystal size decreases (Fig. 3) indicating that the ferromagnetic ground state is favoured in nanocrystals. In fact, the suppression of $\mathrm{T}_{\mathrm{N}}$ has been observed in synthesized orthorhombic MnP nanorods with their growth direction along the $b$ axis and a small diameter $(\sim 5 \mathrm{~nm}),{ }^{14}$ which corresponds to an extreme confinement of the screw structure along the hard axis $a$. In the MnP embedded clusters and polycrystalline films reported here, with larger crystal sizes $(15-40 \mathrm{~nm}), \mathrm{T}_{\mathrm{N}}$ is observed to increase significantly.

The reported hydrostatic or uniaxial strain effects in bulk $\mathrm{MnP},{ }^{15,16}$ do not offer a straightforward explanation for the observed variations of $\mathrm{T}_{\mathrm{C}}$ and $\mathrm{T}_{\mathrm{N}} \cdot{ }^{7}$ However, it is reasonable to suppose that $J_{1}$ and $J_{2}$ may vary in nanocrystals due to the important weight of the surface Mn compared to bulk. Within this framework, we have estimated the dependence of both transition temperatures on the strength of both coupling

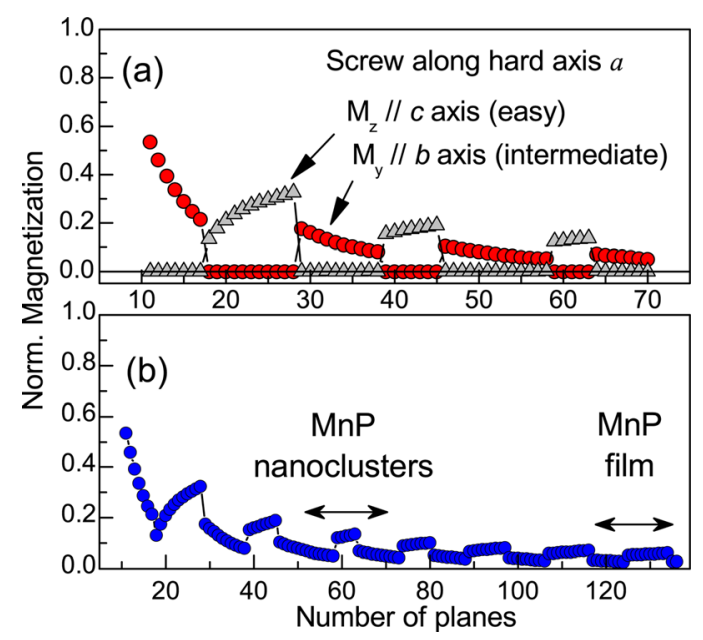

FIG. 3. (Color online) Magnetization normalized to the fully ferromagnetic value vs. the number of planes in the grain (a) along $b$ direction (red circles) and along $c$ (grey triangles). (b) Modulus of the normalized magnetization. $\mathrm{N}=10$ corresponds to $\mathrm{ca} .3 \mathrm{~nm}$. 


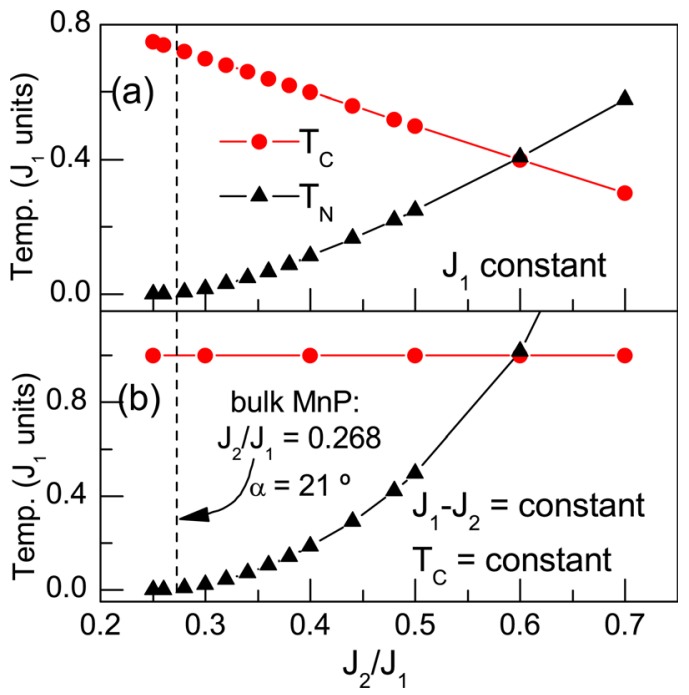

FIG. 4. (Color online) Evolution of $\mathrm{T}_{\mathrm{C}}$ and $\mathrm{T}_{\mathrm{N}}$, with the absolute value of $\mathrm{J}_{2} / \mathrm{J}_{1}$ ( $\mathrm{J}_{1}$ ferro- and $\mathrm{J}_{2}$ antiferro-magnetic coupling constants) in bulk MnP for different conditions: (a) $\mathrm{J}_{1}=$ constant and (b) $\left(\mathrm{J}_{1}-\mathrm{J}_{2}\right)=$ constant.

parameters. In an infinite crystal, the minimization of the energy per plane $E=-J_{1} \cos (\alpha)+J_{2} \cos (2 \alpha)$ (taking $J_{2}>0$ ) yields $\cos (\alpha)=J_{1} /\left(4 J_{2}\right) .{ }^{12}$ Thus, the screw phase $(\alpha \neq 0)$ occurs for $J_{2} / J_{1}>1 / 4$ with an energy $E_{\text {min }}=-J_{1}(\gamma+1 / 8 \gamma)$, where $\gamma=J_{2} / J_{1}$, which deepens as $J_{2} / J_{1}$ increases. Since the energy of the ferromagnetic state $(\alpha=0)$ is $E_{F M}=J_{2}-J_{1}, \mathrm{~T}_{\mathrm{C}}$ is directly related to $J_{2}-J_{1}$, whereas $\mathrm{T}_{\mathrm{N}}$ is related to the difference: $E_{\text {min }}-E_{F M}$. Even if the model is oversimplified, the predicted tendencies for the transition temperatures of both the FM and screw phases are expected to be reliable.

Fig. 4 presents the evolution of $\mathrm{T}_{\mathrm{C}}$ and $\mathrm{T}_{\mathrm{N}}$ as a function of the parameters $J_{1}$ and $J_{2}$. In the upper panel, the $J_{2} / J_{1}$ ratio increases while $\mathrm{J}_{1}$ is fixed, therefore only $\mathrm{J}_{2}$ increases. In this case, close to the $J_{2} / J_{1}$ bulk value for $\mathrm{MnP}(0.268), \mathrm{T}_{\mathrm{C}}$ and $\mathrm{T}_{\mathrm{N}}$ vary in opposite directions; $\mathrm{T}_{\mathrm{N}}$ varying more slowly, as it occurs when applying hydrostatic or uniaxial pressures. For $\mathrm{J}_{2} / \mathrm{J}_{1} \leq 1 / 4$, the system becomes ferromagnetic and $\mathrm{T}_{\mathrm{N}}$ as well as $\alpha$ are zero. When $\left(J_{1}-J_{2}\right)$ is maintained constant and, therefore, $\mathrm{T}_{\mathrm{C}}$ is constant (lower panel) by increasing the ratio $J_{2} / J_{1}, \mathrm{~T}_{\mathrm{N}}$ increases. Therefore, the combination of a constant $\mathrm{T}_{\mathrm{C}}$ and an increase of $\mathrm{T}_{\mathrm{N}}$ observed for the films with grains in the nanometric range can be explained within this model by an increase of both the ferromagnetic and antiferromagnetic couplings, most probably due to the different environment of $\mathrm{Mn}$ at the outmost shell of the grains.

The observed coercive fields are high (above $2 \mathrm{kOe}$ ) but lower than the field corresponding to a single domain coherent rotation with the anisotropy constants of bulk MnP. ${ }^{7}$ The discussed changes in the magnetic structure and magnetic interactions are also providing a lower energy mechanism for the magnetization rotation. The nucleation of helical regions at the grain surface, which is favoured by the antifer- romagnetic coupling compared to an ordinary ferromagnetic system, may be propagated to the grain requiring a much lower energy, i.e., lower coercive fields, than bulk values of a coherent reversal.

From this model, one can conclude that the observed low temperature magnetism of $\mathrm{MnP}$ nanocrystals is related to their nanometric size and to surface effects. Model simulations show that the screw magnetic structure is altered by the finite size of the grains giving rise to a net ferromagnetic moment arising from the outmost five or six Mn planes. Since the structure inside the grains seems unchanged compared to bulk while the surface is subject to strain and disorder, it is reasonable to locate the major changes in a shell of a few atomic planes with different magnetic interactions, $\mathrm{J}_{1}$ and $\mathrm{J}_{2}$, which explains the observed large increase of $\mathrm{T}_{\mathrm{N}}$ while maintaining $\mathrm{T}_{\mathrm{C}}$ almost unchanged. Therefore, local changes in ferromagnetic and antiferromagnetic coupling constants at the surface of the grains, combined with effects in the magnetic structure related to the nanometric size, are postulated at the origin of the differences in the low temperature magnetic behaviour of MnP nanocrystals as compared to powder or single crystals.

We acknowledge the financial support from the Spanish Ministry MICINN (MAT2009-08786, MAT-2008-06517, and CSD2009-00013), from "Comunidad de Madrid" (No. S2009/MAT-1756), and by the Natural Sciences and Engineering Research Council of Canada (NSERC), the infrastructure support of the RQMP (Québec), and the technical assistance of Joël Bouchard.

${ }^{1}$ T. Dietl, Nature Mater. 9, 965 (2010).

${ }^{2}$ J. M. D. Coey, M. Venkatesan, and C. B. Fitzgerald, Nature Mater. 4, 173 (2005).

${ }^{3}$ A. Espinosa, M. García-Hernández, N. Menéndez, C. Prieto, and A. de Andrés, Phys. Rev. B 81, 064419 (2010).

${ }^{4}$ G. A. Prinz, Science 282, 1660 (1998).

${ }^{5}$ A. Gabriel Monette, C. Lacroix, S. Lambert-Milot, V. Boucher, D. Ménard, and S. Francoeur, J. Appl. Phys. 107, 09A949 (2010).

${ }^{6}$ S. Lambert-Milot, C. Lacroix, D. Ménard, R. A. Masut, P. Desjardins, M. García-Hernández, and A. de Andrés, J. Appl. Phys. 104, 083501 (2009).

${ }^{7}$ A. de Andrés, A. Espinosa, C. Prieto, M. García-Hernández, R. RamírezJiménez, S. Lambert-Milot, and R. A. Masut. J. Appl. Phys. 109, 113910 (2011).

${ }^{8}$ S. Rundqvist and P. C. Nawapong, Acta Chem. Scand. 19, 1006 (1965).

${ }^{9}$ E. E. Huber and D. H. Ridgley, Phys. Rev. 135, A1030 (1964).

${ }^{10}$ M. S. Reis, R. M. Rubinger, N. A. Sobolev, M. A. Valente, K. Yamada, K. Sato, Y. Todate, A. Bouravleuv, P. J. von Ranke, and S. Gama, Phys. Rev. B 77, 104439 (2008).

${ }^{11}$ C. C. Becerra, H. J. Brumatto, and N. F. Oliveira, Phys. Rev. B 54, 15997 (1996).

${ }^{12}$ An AFM constant $\left(J_{2}\right)$ is negative, however here we take both $J_{1}$ and $J_{2}$ as positive, and explicitly incorporate the sign in the Hamiltonian.

${ }^{13}$ A. Zieba, M. Slota, and M. Kucharczyk, Phys. Rev. B 61, 3435 (2000).

${ }^{14}$ K. A. Gregg, S. C. Perera, G. Lawes, S. Shinozaki, and S. L. Brock, Chem. Mater. 18, 879 (2006).

${ }^{15}$ E. Hirahara, T. Suzuki, and Y. Matsumura, J. Appl. Phys. 39, 713 (1968).

${ }^{16}$ E. Iwata, J. Sci. Hiroshima Univ., Ser. A-2 33, 1 (1969). 